\title{
Laser-Driven Recollisions Generalized to Relativistic Energies
}

\author{
M. Verschl \\ Max-Planck-Institut für Kernphysik, Saupfercheckweg 1, 69117 Heidelberg, Germany \\ e-mail: mario.verschl@mpi-hd.mpg.de \\ Received October 31, 2007
}

\begin{abstract}
The important process of laser-driven recollisions, where electrons are accelerated by strong laser fields and return to their parent ions, breaks down if the laser intensities imply relativistic electron dynamics. In this case, the Lorentz force drags the electrons away in the laser propagation direction, which inhibits recollisions. Here, a variety of schemes are discussed and compared which generalize the concept of recollisions to the relativistic regime. Additional static electric fields, antisymmetric initial states, and tailored laser pulses are suitable for weakly to moderately relativistic energies, whereas standing waves, preaccelerated ions, positronium, and counterpropagating consecutive pulses allow for recollisions up to the highly relativistic regime.
\end{abstract}

PACS numbers: 41.75.Jv, 34.80.Kw, 41.75.Ht

DOI: $10.1134 / \mathrm{S} 1054660 \mathrm{X} 08050095$

\section{INTRODUCTION}

Laser intensities have increased over many orders of magnitude during the past few decades. The effects of laser-matter interactions range from linear over nonlinear optics to relativistic electron dynamics. The basis for a variety of applications is the important process of laser-driven recollisions. Ions or molecules are, first, ionized by an intense laser field, then the freed electrons are guided and accelerated by the laser electric field, and, finally, they approach the parent ion where they recollide. The ionization usually results from a tunneling process. A potential barrier is formed by the superposition of the laser electric field and the Coulomb field of the ion. After electrons have tunneled out of this barrier, they are at rest. Besides the laser parameters, the energy gain before recollision depends on the laser phase at which the tunneling occurs. The collision energy can be released by the further ionization of the core (nonsequential double ionization), the excitation of the ion, or the emission of radiation. For reviews of the physics of atoms in laser fields, see, e.g., [1-8].

The spectrum of emitted radiation mostly consists of high harmonics (high-harmonic generation). These are created when the matter wave of the tunneled wavepacket returns and interferes with the part of the wavepacket still bound to the ion $[1,9]$. By means of this self-interference, an oscillating charge density is formed which radiates. The whole process repeats with every half laser cycle and, consequently, the emitted radiation interferes and produces a spectrum of odd harmonics. There is an optimum instant of tunneling which maximizes the energy of the recolliding electron. Consequently, the emitted spectrum has a cutoff whose energy is given by the sum of the kinetic energy and the ionization energy.

There are various applications of high harmonics. They can be used as an ultraviolet, coherent light source
$[10,11]$, for probing molecular dynamics $[12,13]$, or for the imaging of molecular orbits [14]. With the superposition of the parts of the harmonic spectrum, single attosecond pulses or attosecond pulse trains can be created [15-17], which allow for the time-resolved observation of processes below the femtosecond time scale.

The energy of recollisions increases with higher laser intensities. However, at some point, the effect of the Lorentz drift becomes important, which suppresses recollisions $[3,7]$. For intensities where the electron dynamics remains nonrelativistic (the velocity $V$ is small compared to the speed of light $c$ ), the force on the electron due to the magnetic field is small:

$$
\mathbf{F}=\mathbf{E}+\frac{\mathbf{v} \times \mathbf{B}}{c} \approx \mathbf{E} .
$$

Note that atomic units are employed throughout this article, i.e., additional to Gaussian units, Planck's constant $\hbar$, the electron mass $m$, and the unit charge $e$ are equal to unity. In these units, the electric and magnetic fields of a propagating plane wave have equal modulus, which means that the force of the magnetic field is suppressed by the factor $v / c$ with respect to the electric field. The electron dynamics is, therefore, dominated by an oscillation in the polarization direction. For higher laser intensities on the order $I \sim 10^{17} \mathrm{~W} / \mathrm{cm}^{2}$ with wavelengths in the visible range, the electron is pushed in the direction orthogonal to both the direction of motion and the magnetic field, which causes the electron to drift in the laser propagation direction. A quantitative description of the Lorentz drift is given in Section 2.

Relativistic recollision energies, which would be required to create coherent $\mathrm{X}$ rays, to excite nuclei or to create pairs are not achievable with this simple recollision scheme. Ideas to generalize the concept of recolli- 
sions to relativistic energies are summarized in this article.

Schemes reducing the drift and, thus, allowing for higher laser intensities are introduced in Section 3. The ideas comprise additional electric fields compensating the magnetic field force [18] and antisymmetric initial states, where the electrons are emitted with an initial momentum directed against the drift $[20,21]$. Furthermore, optimized laser pulses with minimal drift [22, 23 ] and preaccelerated ions, which see Doppler-shifted laser pulses, are discussed [24, 25].

In Section 4, schemes are presented which are easily extended to very high laser intensities without involving additional complications. These schemes are, therefore, particularly suitable for highly relativistic recollisions. Positronium is an appropriate bound system for relativistic recollisions since both particles drift at the same pace [26, 27]. Moreover, standing waves can be applied where the Lorentz drift is circumvented [2831] or two consecutive laser pulses, where the drift of the first pulse is compensated by the second one $[32,33]$.

A summary of the different schemes is given in Section 5, where they are also compared with respect to their most important features like the applicable laser intensity or experimental issues.

\section{DESCRIPTION OF THE LORENTZ DRIFT}

It is convenient to discuss the effect of the Lorentz drift for free laser-driven electrons. After ionization, the effect of the electric field of the core can often be neglected, especially for strong laser fields (strong field approximation [7]), such that considering a free electron is a useful approximation to describe the Lorentz drift. The classical relativistic equations of motion can be solved for a plane laser field [34]. With the laser wave linearly polarized in the $x$ and propagating in the $z$ direction, one finds the following implicit solution:

$$
\begin{gathered}
x=-\frac{1}{\omega c} \int_{0}^{\varphi} A\left(\varphi^{\prime}\right) d \varphi^{\prime}, \\
y=0, \\
z=\frac{1}{2 \omega c^{3}} \int_{0}^{\varphi} A^{2}\left(\varphi^{\prime}\right) d \varphi^{\prime}, \\
\gamma=1+\frac{1}{2 c^{4}} A^{2}, \\
\varphi \equiv \omega(t-z / c),
\end{gathered}
$$

where $A$ denotes the vector potential of the laser field and $\varphi$ is the laser phase seen by the electron. Initially, the electron is assumed to be at rest and placed at the origin. The electron energy is given by the relativistic $\gamma$
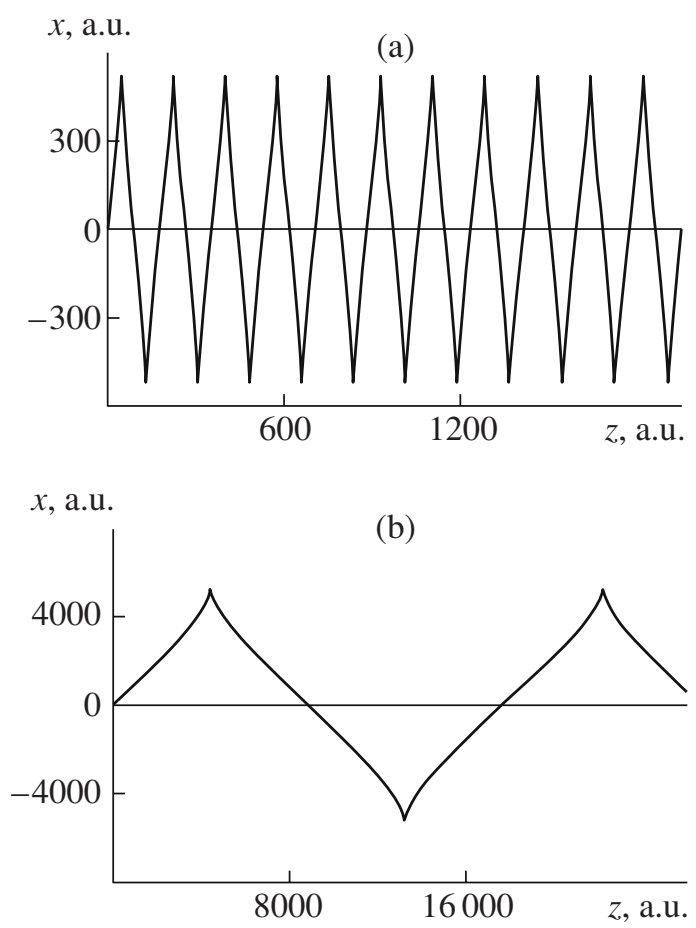

Fig. 1. The electron trajectories in a sinusoidal propagating plane laser wave are depicted for the wavelength $\lambda=$ $800 \mathrm{~nm}$ and the intensities $I=$ (a) $10^{17}$ and (b) $10^{19} \mathrm{~W} / \mathrm{cm}^{2}$. It is seen that the drift in the propagation direction becomes more important with respect to the oscillation in the polarization direction for an increasing laser intensity.

factor (2d). Figure 1 shows the trajectories for two different laser intensities.

For small drifts $(z \omega / c)^{2} \ll 1$, the expansion in $z \omega / c$ on the right-hand side of Eq. (2c) can be truncated after the first-order term, according to

$$
z \approx \frac{1}{2 \omega c^{3}}\left[\int_{0}^{\omega t} A^{2}\left(\varphi^{\prime}\right) d \varphi^{\prime}-A^{2}(\omega t) \frac{\omega}{c} z\right] .
$$

This can be solved for $z$ to obtain an explicit time dependence for the electron position:

$$
\begin{gathered}
x=-\frac{1}{\omega c}\left[\int_{0}^{\omega t} A\left(\varphi^{\prime}\right) d \varphi^{\prime}-\frac{A(\omega t) \frac{1}{2 c^{4}} \int_{0}^{\omega t} A^{2}\left(\varphi^{\prime}\right) d \varphi^{\prime}}{1+\frac{1}{2 c^{4}} A^{2}(\omega t)}\right], \\
y=0, \\
z=\frac{\frac{1}{2 \omega c^{3}} \int_{0}^{\omega t} A^{2}\left(\varphi^{\prime}\right) d \varphi^{\prime}}{1+\frac{1}{2 c^{4}} A^{2}(\omega t)} .
\end{gathered}
$$




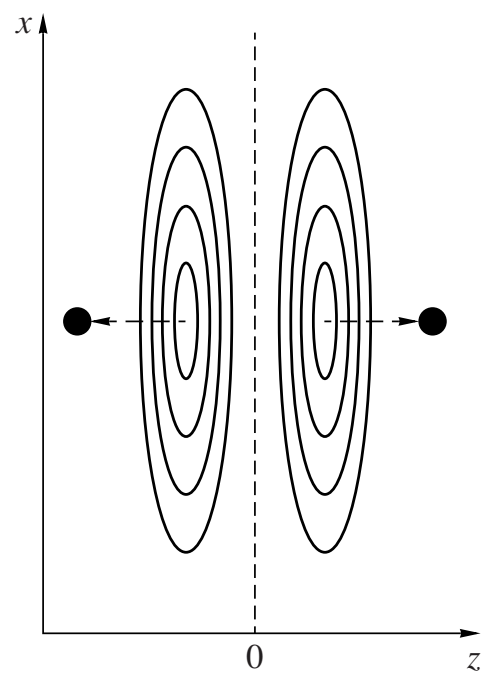

Fig. 2. An antisymmetric atomic or molecular state is sketched which has a nodal plane with a vanishing probability distribution (dashed line). The molecule is aligned with the laser propagation direction $z$. The wave function in momentum space has a similar structure, i.e., electrons freed by ionization have an initial momentum either in the positive or negative $z$ direction. For the latter case, the initial momentum can be employed to cancel the Lorentz drift.

Considering the weakly relativistic limit by neglecting third-order terms of $A / c^{2}$, these expressions further simplify as

$$
\begin{gathered}
x=-\frac{1}{\omega c} \int_{0}^{\omega t} A\left(\varphi^{\prime}\right) d \varphi^{\prime}, \\
y=0, \\
z=\frac{1}{2 \omega c^{3}} \int_{0}^{\omega t} A^{2}\left(\varphi^{\prime}\right) d \varphi^{\prime} .
\end{gathered}
$$

This is equal to the results obtained for nonrelativistic calculations beyond the dipole approximation [35].

\section{RELATIVISTIC RECOLLISIONS DUE TO REDUCED DRIFT}

As discussed in the previous section, recollisions are suppressed by the Lorentz drift if the laser fields become too intense. The schemes introduced in this section have in common that this drift is reduced by some mechanism increasing the applicable intensities to the relativistic regime.

\subsection{Additional Electric Fields}

A straightforward method to compensate for the Lorentz drift is the application of an additional electric field. To counteract the Lorentz force, it has to be directed in the laser propagation direction. The maxi- mum velocity of a weakly relativistic electron oscillating in a laser field with amplitude $E_{0}$ is given by $E_{0} / \omega$ (this follows from (5a) with $E=-\dot{A} / c$ ) and, consequently, the maximum force on account of the magnetic field is given by $E_{0}^{2} /(\omega c)$ (see Eq. (1)). Since this is the maximum force, the averaged counteracting field has to be smaller than that. A two-dimensional model has been employed [18] to numerically simulate the electron dynamics of an $N^{6+}$ ion for a laser pulse with frequency $\omega=1$ and the intensity of $I=1.5 \times 10^{18} \mathrm{~W} / \mathrm{cm}^{2}$ (corresponding to the maximum electric field of $E_{0}=$ $6.5 \mathrm{au})$. Without the additional electric field, the electron center of mass follows a trajectory similar to that of a free electron drifting away from the core. If a proper additional electric field $\left(E_{0} /(13.5 \omega c)\right)$ is applied, the electron revisits the core after several oscillations.

From an experimental point of view, it is much more convenient to employ additional laser fields instead of static ones. Since the electric field should not change its direction before the electron has revisited the core, its frequency must be considerably smaller than the other laser field. For the example discussed above, but this time with an oscillating electric field $(\omega=0.25$ and $E=$ $\left.2 E_{0} /(\omega c)\right)$, even two recollisions have been achieved.

In all, additional electric fields have been shown to generalize the nonrelativistic recollision mechanism to weakly relativistic energies where the drift is only a small effect.

\subsection{Antisymmetric States}

Among other parameters like the laser intensity or the frequency, the electron dynamics of laser-driven atoms or molecules depends on the initial bound state. Depending on the initial momentum, a laser-driven electron drifts with respect to the case of an electron initially at rest. The initial momentum is subject to some probability distribution according to the initial state in momentum space. With this picture, the virtues of an antisymmetric initial state concerning the Lorentz drift are understood intuitively: antisymmetric states in atoms or molecules have a nodal plane where the probability density vanishes (see Fig. 2). This feature is also found for the states in momentum space, i.e., the probability distribution of the initial momentum in the direction orthogonal to the symmetry plane is peaked about a positive and a negative value. If the state is aligned in a way that the nodal plane is orthogonal to the laser propagation direction, the electron will drift on account of the initial momentum either in or against the laser propagation direction, i.e., the Lorentz drift is either amplified or partly canceled.

In terms of wavepackets, this behavior is understood intuitively. Following from the classical description of wavepackets as an ensemble of classical trajectories [41], one-half of the probability distribution recollides, 
given the parameters are chosen appropriately, whereas the other half is lost.

In [20], this scheme has been shown to enhance the high-harmonic generation in a two-dimensional $\mathrm{H}_{2}^{+}$ molecule with proper alignment for the intensity $I=$ $1.4 \times 10^{17} \mathrm{~W} / \mathrm{cm}^{2}$ and the wavelength $\lambda=248 \mathrm{~nm}$. As expected, the enhancement vanishes if the molecule is aligned with the polarization direction.

Similar results for antisymmetric atomic states are found in [21]. The applicable laser intensity increases with the ionization energy, i.e., for higher intensities, one can apply tightly bound electrons of highly charged ions. But, due to the corresponding higher widths of the wavepacket in momentum space, spreading will also reduce the efficiency of the recollisions.

\subsection{Tailored Laser Pulses}

The main parameters for the Lorentz drift are clearly the laser intensity and the frequency. But, it can also be influenced by the laser pulse shape. So, the question arises what pulse shape yields minimal drift. To find the answer, an arbitrary periodic laser field is written as the superposition of high harmonics of the laser frequency $\omega$ with unknown amplitudes $c_{k}$. This Fourier series is given by

$$
E(\varphi)=\sum_{k} c_{k} \exp (i k \omega \varphi)
$$

where $\varphi$ denotes the laser phase. Optimized pulses are then constructed by varying the coefficients with the goal of minimizing the drift. The results of this procedure are depicted in Fig. 3. The features of the optimized pulse train can be understood intuitively. First, the laser field strongly decreases after the first ionization pulse followed by a long time span with a weak laser field strength, during which the electron dynamics is nonrelativistic. Consequently, the drift is suppressed. Second, there is a strong and short pulse shortly before rescattering, i.e., the acceleration force is concentrated on a short time interval. Because of this fast energy gain, the drift is reduced.

The calculations in [23] have shown that, for the ground frequency of the laser $\omega=0.1$, an optimized high-harmonic generation in the relativistic regime is achieved for the pulse duration of 50 as and an average intensity of $I=10^{19} \mathrm{~W} / \mathrm{cm}^{2}$.

Intense tailored pulses could be created by spectral filtering of the attosecond-pulse trains generated by laser-foil interactions (sliding mirror) [36]. Due to the limited efficiency of this process, the initial laser intensity has to be up to three orders of magnitude higher than the intensity of the tailored pulses.

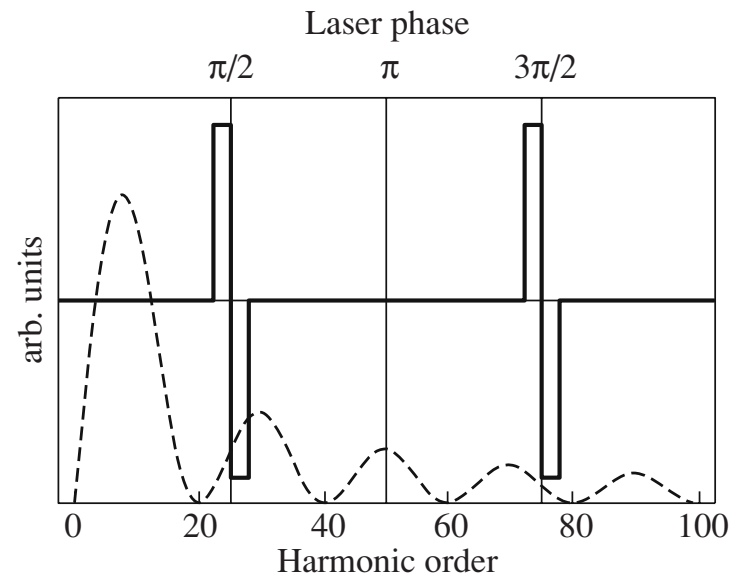

Fig. 3. A pulse train with an optimized shape is shown (bold line) which minimizes the Lorentz drift. The dashed line gives the corresponding spectrum of the pulse train.

\subsection{Preaccelerated Ions}

The relativistic Doppler shift is another means by which the Lorentz drift can be reduced. In this case, laser-driven recollisions occur in ions accelerated to high energies. Consequently, the laser light is more intense and has a shorter wavelength in the rest frame of the ions.

What the laser light looks like in the comoving frame is easily calculated by means of a Lorentz transformation. The ions are moving in the opposite direction of laser propagation with velocity $v$. Since the vector potential of the laser field (the four vector) has only a component in the polarization direction, it remains unchanged by the Lorentz transformation, whereas the laser frequency is Doppler shifted:

$$
\begin{gathered}
A^{\prime}\left(z^{\prime} \omega^{\prime} / c-\omega^{\prime} t^{\prime}\right)=A(z \omega / c-\omega t), \\
\frac{\omega^{\prime}}{\omega}=\sqrt{\frac{1+v / c}{1-v / c}}=\left(\gamma_{i}+\sqrt{\gamma_{i}^{2}-1}\right) \approx 2 \gamma_{i} .
\end{gathered}
$$

The prime denotes the corresponding quantities in the comoving frame. Here, the frequency shift is also given in terms of the $\gamma$ factor $\left(\gamma_{i}\right)$ corresponding to the velocity $v$ of the comoving frame. For the high velocities of interest here $\left(\gamma_{i}^{2} \gg 1\right)$, the approximation in Eq. (7b) applies.

From Eq. (2d) and the invariance of $A$ (see (7a)), it is seen that the maximal electron energy in the comoving frame is independent of the relative velocity $v$. However, according to Eq. (2c), the drift in the laser propagation direction is reduced by the factor $\omega^{\prime} / \omega \approx 2 \gamma_{1}$.

Consequently, the maximum laser energy at which the Lorentz drift is still small enough to allow for recollisions is limited by the achievable kinetic energy of the accelerated ions. With the large-scale accelerator at CERN, energies of $0.4 \mathrm{TeV}$ per unit charge have been 
reached [37]. Applied to $\mathrm{O}^{7+}$, this would accelerate the ions to values of $\gamma_{i} \approx 185$. Recollision energies could, thus, be enhanced by factors of a few hundred. For laser wavelengths of $\lambda=800 \mathrm{~nm}$, the typical intensity of $I \sim$ $10^{17} \mathrm{~W} / \mathrm{cm}^{2}$, where the drift becomes important, could, therefore, be increased up to $I \sim 10^{19} \mathrm{~W} / \mathrm{cm}^{2}$.

\section{RECOLLISION SCHEMES FOR HIGHLY RELATIVISTIC ENERGIES}

The following schemes are more suitable for recollisions with high energies than the ones from the previous section, because the Lorentz drift is not treated as a disturbing effect which can be attenuated or compensated. Other ideas are introduced, where the drift does not occur at all or where it is a constituent of the scheme.

\subsection{Positronium}

Electrons drifting in the laser propagation direction cannot recollide, because the core remains at its position due to its high mass. This problem can be solved by employing positronium atoms, where both electrons and positrons drift at the same velocity. Recollisions can then occur even for highly relativistic laser intensities. However, it has to be considered that the collision energy in the center of momentum frame $E^{\prime}$ is less than the energy in the rest frame (two times the value of Eq. (2d)), especially for high energies. In the comoving frame, the energy of the electron (or positron) is given by

$$
E^{\prime^{2}}=c^{4}+(\gamma \dot{x} c)^{2},
$$

because the momentum in the $z$ direction is zero by definition and the momentum in the $x$ direction is not affected by the Lorentz transformation. By means of Eqs. (2), the following $\gamma$ factor for the electron is, then, found in the comoving frame:

$$
\gamma^{\prime}=\sqrt{1+\frac{1}{c^{4}} A^{2}} .
$$

The total collision energy $2 c^{2} \gamma^{\prime}$ is approximately proportional to $A$ for high energies compared to the quadratic dependence in the rest frame. This means that, in the highly relativistic regime, much energy is lost for kinematic reasons. This problem can partially be solved by means of an additional magnetic field [27], which causes the particles to collide somewhat more headon. For considerable enhancements of the collision energies in the highly relativistic regime, magnetic fields of several hundred Tesla are required.

Furthermore, it needs to be considered that the particles do not necessarily recollide at the instant of maximal energy. The actual energy depends on the laser pulse shape and on the laser phase at the instant of separation.
An experimental requirement to achieve high recollision rates is the availability of a dense positronium gas.

\subsection{Standing Waves}

The Lorentz drift occurring in propagating laser fields can be canceled by a second counterpropagating wave with the same intensity. In this case, the electrons are driven by a standing wave. Both linearly [29-31] and circularly polarized fields [28] can be superimposed to eliminate the drift. However, the electron dynamics differ for the two configurations [38].

In a classical picture, the ideal motion for both setups is a one-dimensional oscillation driven by the electric field. One finds the following analytical solution for this case:

$$
\begin{gathered}
x=-c \int_{0}^{t} \frac{-\frac{1}{c^{2}} A\left(t^{\prime}\right)}{\sqrt{1+\frac{1}{c^{4}} A^{2}\left(t^{\prime}\right)}} d t^{\prime}, \\
\gamma=\sqrt{1+\frac{1}{c^{4}} A^{2}} .
\end{gathered}
$$

It is seen that the energy expressed by the relativistic $\gamma$ factor is equal to the electron energy in the center of momentum frame of laser-driven positronium (compare Eq. (8)), i.e., the collision energy in a standing laser field is also smaller than in a propagating field of the same intensity. Moreover, the recollision energy depends on the laser pulse shape and the laser phase at the instant of ionization.

4.2.1. Linear polarization. Two counterpropagating laser waves of equal amplitudes polarized in the $x$ direction generate the electromagnetic field

$$
\begin{aligned}
& \mathbf{E}=E_{0} \cos (\omega t) \cos (k z) \hat{\mathbf{x}}, \\
& \mathbf{B}=E_{0} \sin (\omega t) \sin (k z) \hat{\mathbf{y}}, \\
& \mathbf{A}=A_{0} \sin (\omega t) \cos (k z) \hat{\mathbf{x}},
\end{aligned}
$$

where the fields propagate in the $\pm z$ direction [42]. It is seen that the magnetic field vanishes in those planes with maximal electric field amplitudes. However, if the electrons do not move exactly in these planes, they are disturbed by the nonvanishing magnetic fields. By means of a straightforward classical analysis, it can be shown that the Lorentz force due to the magnetic field is directed away from the ideal planes causing an instability in the $z$ direction.

Figure 4 shows a laser-driven wavepacket which is constructed by means of a classical Monte Carlo approach (see [38]). Besides the instability, the relativistic effect of time dilation is observed. This is seen for the relativistic case, where wavepacket spreading is 
(a)

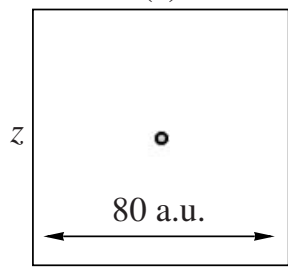

(b)

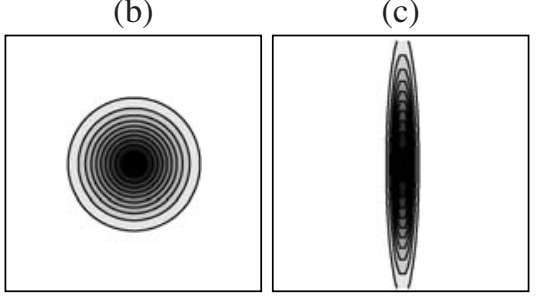

$y$ (a)

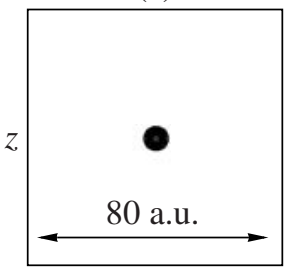

(b)

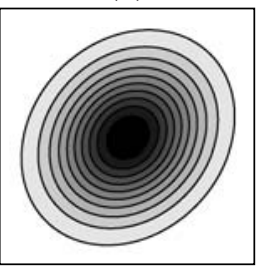

y (c)

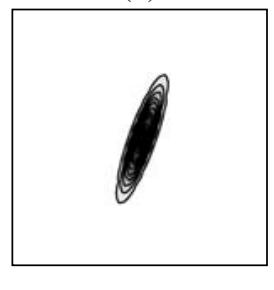

Fig. 5. The probability density of a wavepacket in circularly polarized crossed beams is plotted for the initial time (Fig. (a)) and after two full laser periods ((b) and (c)). The wavepackets are shown for the plane perpendicular to the direction of motion. Figure (b) gives a nonrelativistic example $\left(R \equiv E_{0} / \omega c=A_{0} / c^{2}=0.1\right)$, whereas (c) is the relativistic case of $R=25$ corresponding to the intensity of $7 \times$ $10^{20} \mathrm{~W} / \mathrm{cm}^{2}$ for $\lambda=800 \mathrm{~nm}$. It is seen that spreading is inhibited in the relativistic case on account of time dilation.

electrons away from their parent ions by means of the Lorentz drift, whereas the second pulse drives them back. This idea is briefly summarized in Fig. 6. With different pulse intensities, recollisions can occur when the kinetic energy is maximal. As seen from equations for a relativistic laser-driven electron (2), the energy scales quadratically with the vector potential. For the highly relativistic case, this allows for much higher collision energies than for the schemes with standing waves or positronium (see Eqs. (8) and (10b)). The reason for the higher energy is the fact that the electron moves with the propagating laser pulse and, therefore, the laser frequency is Doppler shifted to lower frequencies, such that the time for acceleration is longer.

Since the electron dynamics is two dimensional, the laser pulses have to be controlled accurately to insure recollisions.

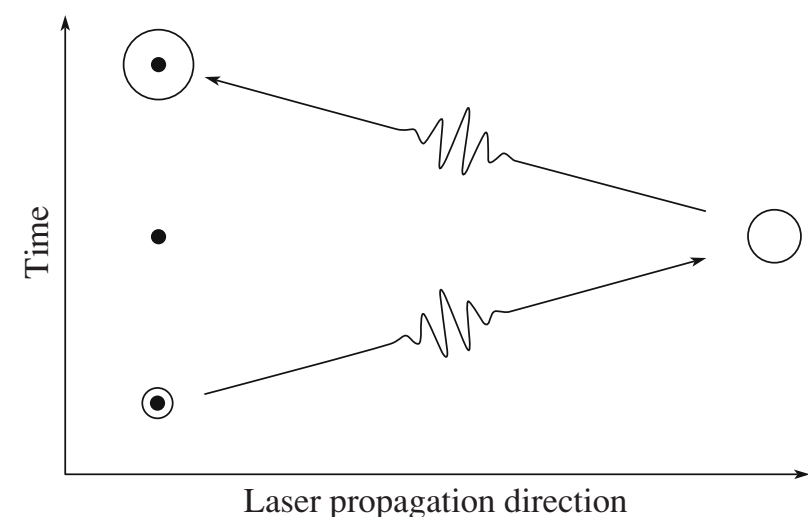

Fig. 6. The Lorentz drift of two intense laser pulses is employed, first, to separate electrons from the core and, second, to bring them back for recollisions. The pulses have to be short in order to reduce the losses of effectivity due to wavepacket spreading. This scheme allows for recollisions at the instant of maximal electron energy. 


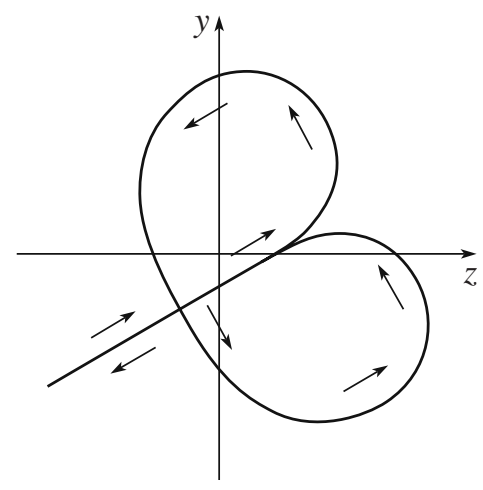

Fig. 7. Here, a trajectory of an electron is depicted which effectively reverses its momentum. The electron is guided by a time-dependent magnetic field perpendicular to the $y$ $z$ plane. The curvature of the trajectory is proportional to the magnetic field, which results in a magnetic double pulse requiring the proper timing and amplitudes. Additional electric fields induced by the varying magnetic field also have to be considered.

For the calculations, the laser intensity is assumed to be so high that the ionization occurs immediately (over the barrier ionization) and the electron can be considered as a free particle.

The probability $R$ for some reaction in a gas sample depends on the gas density $n$, the cross section of the reaction volume $S$ (perpendicular to the propagation direction $z$ ), and on the total reaction cross section $\sigma$, according to

$$
R=n S \sigma f .
$$

The function $f$ depends on the initial electron wavepacket and on the laser pulse shape. It is found (see [32]) that the reaction rate decreases with higher laser energies and longer pulses, since this increases the duration of the whole process and, with this, the spreading time. Concerning the initial width in momentum space, there is an ideal value maximizing the yield. This behavior is understood with the fact that the initial width in momentum space affects both the initial spatial width and the rapidness of spreading.

As an example, consider the creation of electronpositron pairs in a sample of cesium atoms. With $n=$ $10^{15} \mathrm{~cm}^{-3}, S=10 \mu \mathrm{m}$, and a repetition rate of $v=$ $100 \mathrm{~Hz}$, it is found that one event is expected after $37 \mathrm{~min}$ for $\sigma=6.3 \times 10^{-25} \mathrm{~cm}^{2}$. This number for the cross section $\sigma$ is found for the collision energy of $100 \mathrm{MeV}$, which corresponds to a laser intensity of about $10^{21} \mathrm{~W} \mathrm{~cm}^{-2}$. To keep spreading as small as possible, short laser pulses of a few cycles have been chosen.

4.3.1. Refocused recollisions. A general problem which reduces the reaction rates of recollisions is wavepacket spreading. The previous scheme can be extended to address this problem. An additional short and intense magnetic field, which is applied after the first laser pulse, can be employed to reverse the spreading dynamics such that the wavepacket contracts again.

Spreading can be understood classically as a consequence of uncertain initial conditions. For example, if the electron has some nonvanishing initial momentum, it will be found at a different position than for the case at which it is initially at rest. The longer the propagation time, the greater the distances between trajectories of different initial momenta will have grown. This is the essence of wavepacket spreading. The distribution of the initial conditions follows from the initial quantummechanical state.

With this model in mind, it can be intuitively understood that a spreading wavepacket contracts again if the momentum of the electron is reversed. Any trajectory with nonvanishing initial momentum will-after the momentum reversal-approach the one corresponding to the case of the electron initially being at rest. The distances between the trajectories then decrease. The required momentum reversal can be achieved in two dimensions by means of a magnetic field as shown in Fig. 7. Because refocusing works only for the directions perpendicular to the magnetic field, it has to be aligned in a way that the spreading direction is parallel to the velocity of the recolliding electron.

This scheme requires very short and very strong magnetic field pulses. Pulses in the picosecond regime on the order of a few Tesla have been created by means of relativistic electron bunches $[39,40]$. With pulses of a similar strength and duration, refocusing could be demonstrated, but effective recollisions require stronger and shorter pulses [33]. Pulses of $600 \mathrm{~T}$ and a duration of $100 \mathrm{fs}$ allow for recollisions of several hundred megaelectronvolts with effectivities corresponding to collisions of Gaussian wavepackets with spatial widths of a few atomic units. Whereas this scheme allows for efficient collisions in the highly relativistic regime, the experimental feasibility is challenging.

\section{CONCLUSIONS}

It has been shown that the energies of laser-driven recollisions are limited by the Lorentz drift, i.e., additionally to the oscillation of the direction of laser polarization, the electrons are pushed in the propagation direction. This relativistic effect can inhibit recollisions with the parent ion. Different approaches to enable recollisions with relativistic energies have been presented and discussed. They have different features in terms of achievable collision energies, experimental complexity, or effectivity, which are summarized in Tables 1 and 2.

Some approaches are aimed at influencing the drift to reach some higher collision energies, but, still, an upper limit remains. For intensities on the order $I$ $10^{18} \mathrm{~W} / \mathrm{cm}^{2}$ (in the following, the laser light is always assumed to be in the visible range), the Lorentz drift can, e.g., be tackled with an additional electric field 
Table 1. Some features of the relativistic recollisions schemes based on suppressing the Lorentz drift are given. These schemes appear appropriate for the moderately relativistic regime. The intensities refer to wavelengths in the visible regime

\begin{tabular}{|c|c|c|c|}
\hline Scheme & Applicable intensity & Experimental implementation & Characteristics \\
\hline $\begin{array}{l}\text { Additional electric } \\
\text { fields }\end{array}$ & $I \sim 10^{18} \mathrm{~W} / \mathrm{cm}^{2}$ & $\begin{array}{l}\text { Additional strong electric field } \\
\text { created with a low-frequency laser }\end{array}$ & $\begin{array}{l}\text { Applies in the weakly relativistic } \\
\text { regime, where drift is a rather small } \\
\text { effect }\end{array}$ \\
\hline $\begin{array}{l}\text { Antisymmetric } \\
\text { initial states }\end{array}$ & $\begin{array}{l}\text { Laser intensity depends } \\
\text { on the ionization potential }\end{array}$ & $\begin{array}{l}\text { Alignment of the electronic orbit- } \\
\text { als required }\end{array}$ & $\begin{array}{l}\text { Applies to molecules } \\
\text { and atoms/ions }\end{array}$ \\
\hline $\begin{array}{l}\text { Tailored laser } \\
\text { pulses }\end{array}$ & $\begin{array}{l}\text { Optimized relativistic } \\
\text { high-harmonic generation } \\
\text { for } I \sim 10^{19} \mathrm{~W} / \mathrm{cm}^{2}\end{array}$ & $\begin{array}{l}\text { Intense high harmonics needed } \\
\text { for tailored pulses can be realized } \\
\text { with laser-foil interactions }\end{array}$ & $\begin{array}{l}\text { Intensity losses due to the creation } \\
\text { of the pulses is expected on the } \\
\text { order of up to three magnitudes }\end{array}$ \\
\hline Preaccelerated ions & $\begin{array}{l}I \sim 10^{19} \mathrm{~W} / \mathrm{cm}^{2} \text { with current } \\
\text { technology }\end{array}$ & $\begin{array}{l}\text { Ions need to be accelerated } \\
\text { to energies much higher than their } \\
\text { rest energy }\end{array}$ & $\begin{array}{l}\text { Electron kinetic energy in the } \\
\text { comoving frame remains the same, } \\
\text { but the drift is reduced }\end{array}$ \\
\hline
\end{tabular}

Table 2. This table gives a comparison of the schemes appropriate for recollision in the highly relativistic regime. Since the collision energy is not limited by the laser intensity, the $\gamma$ factor of the electron is given as a function of the laser vector potential $A$

\begin{tabular}{|c|c|c|c|}
\hline Scheme & Electron energy & Experimental implementation & Characteristics \\
\hline $\begin{array}{l}\text { Positronium (alterna- } \\
\text { tively, with an addi- } \\
\text { tional magnetic field) }\end{array}$ & $\begin{array}{l}\text { Effective energy } \gamma=\sqrt{1+A^{2} / c^{4}} \text {; } \\
\text { higher energy with an additional } \\
\text { magnetic field }\end{array}$ & $\begin{array}{l}\text { Dense positronium gas } \\
\text { required for high reaction } \\
\text { rates; the additional magnetic } \\
\text { field has to be extremely strong }\end{array}$ & $\begin{array}{l}\text { Effective collision energy } \\
\text { is reduced due to the drifting } \\
\text { center of momentum }\end{array}$ \\
\hline $\begin{array}{l}\text { Standing waves (lin- } \\
\text { ear polarization) }\end{array}$ & $\begin{array}{l}\text { Energy limited by decreasing } \\
\text { reaction rates; } \gamma=\sqrt{1+A^{2} / c^{4}}\end{array}$ & $\begin{array}{l}\text { Superposition of intense coun- } \\
\text { terpropagating waves }\end{array}$ & $\begin{array}{l}\text { Recollisions occurring only } \\
\text { at nodal planes of the laser } \\
\text { wave; less electron energy } \\
\text { than in propagating laser } \\
\text { fields of an equal intensity }\end{array}$ \\
\hline $\begin{array}{l}\text { Standing waves (cir- } \\
\text { cular polarization) }\end{array}$ & $\gamma=\sqrt{1+A^{2} / c^{4}}$ & $\begin{array}{l}\text { Superposition of circularly } \\
\text { polarized light }\end{array}$ & $\begin{array}{l}\text { Electron current density } \\
\text { increases with stronger laser } \\
\text { fields; less electron energy } \\
\text { than in propagating laser } \\
\text { fields of an equal intensity }\end{array}$ \\
\hline $\begin{array}{l}\text { Consecutive laser } \\
\text { pulses }\end{array}$ & $\gamma=1+A^{2} /\left(2 c^{4}\right)$ & $\begin{array}{l}\text { Accurate control of the laser } \\
\text { pulse shape required }\end{array}$ & $\begin{array}{l}\text { Recollision at the instant } \\
\text { of maximal kinetic energy; } \\
\text { rather low reaction rates }\end{array}$ \\
\hline $\begin{array}{l}\text { Refocused recolli- } \\
\text { sions }\end{array}$ & $\gamma=1+A^{2} /\left(2 c^{4}\right)$ & $\begin{array}{l}\text { Strong and extremely short } \\
\text { magnetic fields required; } \\
\text { extremely high precision } \\
\text { needed }\end{array}$ & $\begin{array}{l}\text { Effective recollisions with } \\
\text { maximal kinetic energy }\end{array}$ \\
\hline
\end{tabular}

directed against the laser propagation direction. The strong fields required can be realized with a laser. Its frequency must be smaller than the one of the driving field, such that the direction of the counteracting field does not reverse too quickly. This approach can be applied if the drift is only a small correction of the dominant oscillating electronic motion. However, wavepacket spreading is faster for a strongly bound electron, which reduces the efficiency of recollisions.

With antisymmetric initial states of molecules or atoms, electrons freed by ionization have either a positive or a negative initial momentum, with respect to the symmetry axis of the bound state, causing an additional drift. With the proper alignment of the molecules or the atomic states, this motion can be employed to cancel the Lorentz drift. The initial momentum, and, therefore, the applicable intensity, depends on how tightly the electron is bound.

Laser pulses can be tailored in a way that the drift is minimized. This is achieved by maximizing the time of nonrelativistic dynamics and minimizing the time of the acceleration to high energies. Moderately relativistic energies of $I \sim 10^{19} \mathrm{~W} / \mathrm{cm}^{2}$ could be realized, but the creation of the intense high-harmonic spectrum, which 
is required to generate the optimized pulses, is a challenging task. By means of the realization with laserfoil interactions, most of the initial intensity would be lost for the creation of the optimized pulses.

In the rest frame of strongly preaccelerated ions, optical laser light has a shorter wavelength at a much higher intensity. This reduces the drift and, therefore, allows for recollisions with higher energies. However, for significant enhancements, the ions need to be accelerated to energies much higher than their resting mass, which requires large-scale accelerating facilities.

These schemes, which are aimed at reducing or compensating the Lorentz drift, seem to be suitable for recollisions in the moderately relativistic regime, i.e., up to $I \sim 10^{19} \mathrm{~W} / \mathrm{cm}^{2}$. Higher intensities may be possible, but with additional complications, such as the need for higher energies to preaccelerate ions or working with strongly bound electrons, which implies a reduced efficiency due to quick wavepacket spreading.

If much higher collision energies in the strongly relativistic regime are required, other schemes are more convenient. For example, in standing-wave configurations, the optimum trajectories remain one dimensional. However, there are differences in the stability of these trajectories. For linearly polarized light, an instability in the propagation direction occurs, which reduces the current density of the returning electron at the nuclear position. This problem, which reduces the probability of collisions, does not occur if the standing wave consists of circularly polarized light. In this case, the spreading reduces on account of time dilation, which even enhances the probability of collisions. Furthermore, recollisions are not constrained to nodal planes as in the case of linear polarization.

With positronium employed for recollisions, the Lorentz drift still occurs, but both particles drift with equal velocities. Although recollisions occur for an arbitrary laser intensity, the collision energy in the center of momentum frame is significantly smaller than the kinetic energy in the rest frame. However, the effective collision energy is on the same order as for the schemes with standing waves. Higher energies can be reached with additional strong magnetic fields.

Recollisions with maximal kinetic energies can be obtained with two consecutive counterpropagating laser pulses. The system can be tuned in such a way that the electrons recollide when they reach the highest energy, which they can achieve in propagating laser fields. Due to the long duration of the process, the reaction rate is lower than for the other schemes. However, with the concept of magnetic refocusing, spreading can be reversed to enable effective recollisions. These schemes require precise control of the laser fields to enable recollisions. For the approach including magnetic refocusing, strong and short magnetic fields are needed, whose creation is still an experimental challenge.
As for these high-energy recollision schemes, the simplest setup is to work with positronium. However, high reaction rates require the availability of a highdensity positronium gas. Higher reaction rates can be achieved with circularly polarized standing waves with similar collision energies. Better scaling of the collision energy with the laser intensity can be achieved with consecutive laser pulses, but at the cost of a rather low reaction probability. Both efficient and highly energetic collisions would be reached with refocused recollisions, but also require considerable experimental advances.

Concluding, the concept of recollisions can be generalized to relativistic energies, where several schemes are available for different requirements such as the collision energy or the experimental effort. Therefore, one has to choose the scheme which is most appropriate for the specific application.

\section{REFERENCES}

1. W. Becker et al., Adv. At. Mol. Opt. Phys. 48, 35 (2002).

2. A. L'Huillier, K. Schäfer, and K. Kulander, J. Phys. B 24, 3315 (1991).

3. D. Milosevic and F. Ehlotzky, Adv. At. Mol. Opt. Phys. 49, 373 (2003).

4. C. Joachain, M. Dörr, and N. Kylstra, Adv. At. Mol. Opt. Phys. 42, 225 (2000).

5. M. Protopapas, C. H. Keitel, and P. L. Knight, Rep. Prog. Phys. 60, 389 (1997).

6. C. H. Keitel, Contemp. Phys. 42, 353 (2001).

7. Y. I. Salamin et al., Phys. Rep. 427, 41 (2006).

8. G. A. Mourou, T. Tajima, and S. V. Bulanov, Rev. Mod. Phys. 78, 309 (2006).

9. W. Becker et al., Phys. Rev. A 56, 645 (1997).

10. C. Spielmann et al., Science 278, 661 (1997).

11. Z. Chang et al., Phys. Rev. Lett. 79, 2967 (1997).

12. M. Lein, Phys. Rev. Lett. 94, 053004 (2005).

13. S. Baker et al., Science 312, 424 (2006).

14. J. Itatani et al., Nature 432, 867 (2004).

15. P. M. Paul et al., Science 292, 1689 (2001).

16. M. Hentschel et al., Nature 414, 509 (2001).

17. A. Scrinzi et al., J. Phys. B 39, R1 (2006).

18. R. Fischer, A. Staudt, and C. H. Keitel, Comput. Phys. Commun. 157, 139 (2004).

19. M. Gajda et al., Phys. Rev. A 46, 1638 (1992).

20. R. Fischer, M. Lein, and C. H. Keitel, Phys. Rev. Lett. 97, 143901 (2006).

21. R. Fischer, M. Lein, and C. H. Keitel, J. Phys. B 40, F113 (2007).

22. M. Klaiber, K. Z. Hatsagortsyan, and C. H. Keitel, Phys. Rev. A 74, 051803(R) (2006).

23. M. Klaiber, K. Z. Hatsagortsyan, and C. H. Keitel, Phys. Rev. A 75, 063413 (2007).

24. G. R. Mocken and C. H. Keitel, J. Phys. B 37, L275 (2004).

25. C. C. Chirila et al., Phys. Rev. Lett. 93, 243603 (2004). 
26. B. Henrich, K. Z. Hatsagortsyan, and C. H. Keitel, Phys. Rev. Lett. 93, 013601 (2004).

27. K. Z. Hatsagortsyan, C. Müller, and C. H. Keitel, Europhys. Lett. 76, 29 (2006).

28. N. Milosevic, P. B. Corkum, and T. Brabec, Phys. Rev. Lett. 92, 013002 (2004).

29. N. Kylstra et al., Phys. Rev. Lett. 85, 1835 (2000).

30. V. D. Taranukhin, J. Opt. Soc. Am. B 19, 1132 (2002).

31. J. de Aldana and L. Roso, J. Opt. Soc. Am. B 19, 1467 (2002).

32. M. Verschl and C. H. Keitel, J. Phys. B 40, F69 (2007).

33. M. Verschl and C. H. Keitel, Europhys. Lett. 77, 64004 (2007).

34. L. D. Landau and E. M. Lifschitz, The Classical Theory of Fields (Pergamon, Oxford, 1971), p. 112
35. M. Verschl and C. H. Keitel, Laser Phys. 15, 529 (2005).

36. A. S. Pirozhkov et al., Phys. Plasmas 13, 013107 (2006).

37. H. F. Krause et al., Phys. Rev. Lett. 80, 1190 (1998).

38. M. Verschl and C. H. Keitel, Phys. Rev. ST Accel. Beams 10, 024001 (2007).

39. S. Takeyama, T. Osada, and N. Miura, Physica B 346, 576 (2004).

40. Y. Matsuda, Rev. Sci. Instrum. 73, 4288 (2002).

41. This method is known as phase-space averaging; see e.g. [19].

42. This expression can be rewritten as the superposition of two propagating waves by applying the appropriate trigonometric identities. 